\title{
MULTIFRACTAL SPECTRUM IN A SELF-SIMILAR ATTRACTOR IN THE UNIT INTERVAL
}

\author{
IN SOO BAEK
}

ABstract. We study the multifractal spectrum of two dimensionally indexed classes whose members are distribution sets of a self-similar attractor in the unit interval.

\section{Introduction}

Recently multifractal spectrum of distribution set of a self-similar set was investigated in $[1,6,2,10]$. More recently Hausdorff dimension of some union of one-dimensionally indexed distribution sets was also investigated([4]). In this paper, we study multifractal spectrum of the union of some two dimensionally indexed distribution sets. It should be observed that at the first beginning of its study it did not attract its attention for some reason of no necessity. Recently our research $([5])$ in the characterization of distribution sets in the Riesz-NágyTakács singular functions induced us to consider the multifractal spectrum of the union of two dimensionally indexed distribution sets.

\section{Preliminaries}

We denote $F$ a self-similar attractor, which is the attractor of the similarities $f_{1}(x)=a x$ and $f_{2}(x)=b x+(1-b)$ on $I=[0,1]$ with $a>0, b>0$ and $1-(a+b) \geq 0$. If $1-(a+b)=0$, then $F$ satisfies so-called the open set condition with a non-empty bounded open set as $(0,1)$. If $1-(a+b)>0$, then the self-similar attractor $F$ is the self-similar Cantor set $([1,8])$. Let $I_{i_{1}, \ldots, i_{k}}=$ $f_{i_{1}} \circ \cdots \circ f_{i_{k}}(I)$ where $i_{j} \in\{1,2\}$ and $1 \leq j \leq k$. We note that if $x \in F$, then there is $\sigma \in\{1,2\}^{\mathbb{N}}$ such that $\bigcap_{k=1}^{\infty} I_{\sigma \mid k}=\{x\}$ (Here $\sigma \mid k=i_{1}, i_{2}, \ldots, i_{k}$ where $\left.\sigma=i_{1}, i_{2}, \ldots, i_{k}, i_{k+1}, \ldots\right)$. Without confusion, we identify $x \in F$ with $\sigma \in\{1,2\}^{\mathbb{N}}$ where $\bigcap_{k=1}^{\infty} I_{\sigma \mid k}=\{x\}$. In $F=[0,1]$, the end point $x$ of $I_{i_{1}, \ldots, i_{k}}$ can be represented as different codes, that is, $x=\sigma$ and $x=\tau$ with $\sigma \neq \tau$. We disregard these points since they are at most countable, which do not affect the values of dimensions. Let $p \in(0,1)$ and we denote $\gamma_{p}$ a self-similar Borel

Received April 3, 2008.

2000 Mathematics Subject Classification. Primary 28A78, 28A80.

Key words and phrases. Hausdorff dimension, packing dimension, multifractal, distribution set, self-similar attractor. 
probability measure on $F$ satisfying $\gamma_{p}\left(I_{1}\right)=p($ cf. [8]) $\operatorname{dim}(E)$ and $\operatorname{Dim}(E)$ denote the Hausdorff dimension and packing dimension of $E$ respectively([8]). We denote $n_{1}(x \mid k)$ the number of times the digit 1 occurs in the first $k$ places of $x=\sigma$ (cf. [1]). For $r \in[0,1]$, we define the lower(upper) distribution set $\underline{F}(r)(\bar{F}(r))$ containing the digit 1 in proportion $r$ by

$$
\begin{aligned}
& \underline{F}(r)=\left\{x \in F: \liminf _{k \rightarrow \infty} \frac{n_{1}(x \mid k)}{k}=r\right\}, \\
& \bar{F}(r)=\left\{x \in F: \limsup _{k \rightarrow \infty} \frac{n_{1}(x \mid k)}{k}=r\right\} .
\end{aligned}
$$

We call $\{\underline{F}(r): 0 \leq r \leq 1\}$ the lower distribution class and $\{\bar{F}(r): 0 \leq r \leq 1\}$ the upper distribution class. by

Similarly for $r_{1}, r_{2} \in[0,1]$ with $r_{1} \leq r_{2}$, we define a distribution set $F\left[r_{1}, r_{2}\right]$

$$
F\left[r_{1}, r_{2}\right]=\underline{F}\left(r_{1}\right) \cap \bar{F}\left(r_{2}\right) .
$$

We note that our disregarded end points $I_{i_{1}, \ldots, i_{k}}$ in $F=[0,1]$ are only members in $F(0)$ or $F(1)$. If $x \in I_{\tau}$ where $\tau \in\{1,2\}^{k}$, a cylinder $c_{k}(x)$ denotes the fundamental interval $I_{\tau}$ and $\left|c_{k}(x)\right|$ denotes the diameter of $c_{k}(x)$ for each $k=0,1,2, \ldots$

We write $\underline{E}_{\alpha}^{(p)}\left(\bar{E}_{\alpha}^{(p)}\right)$ for the set of points at which the lower(upper) local cylinder density of $\gamma_{p}$ on $[0,1]$ is exactly $\alpha$, so that

$$
\begin{aligned}
& \underline{E}_{\alpha}^{(p)}=\left\{x: \liminf _{k \rightarrow \infty} \frac{\log \gamma_{p}\left(c_{k}(x)\right)}{\log \left|c_{k}(x)\right|}=\alpha\right\}, \\
& \bar{E}_{\alpha}^{(p)}=\left\{x \limsup _{k \rightarrow \infty} \frac{\log \gamma_{p}\left(c_{k}(x)\right)}{\log \left|c_{k}(x)\right|}=\alpha\right\} .
\end{aligned}
$$

We write $\underline{E}_{\alpha}^{(p)} \cap \bar{E}_{\alpha}^{(p)}=E_{\alpha}^{(p)}$ and call it the local dimension set having local cylinder density $\alpha$ by a self-similar measure $\gamma_{p}$. In a self-similar Cantor set $F$, we clearly see that $\underline{E}_{\alpha}^{(p)}\left(\bar{E}_{\alpha}^{(p)}\right)$ is the set of points at which the lower(upper) local dimension of $\gamma_{p}$ on $F$ is exactly $\alpha$, so that

$$
\begin{aligned}
& \underline{E}_{\alpha}^{(p)}=\left\{x: \liminf _{r \rightarrow 0} \frac{\log \gamma_{p}\left(B_{r}(x)\right)}{\log r}=\alpha\right\}, \\
& \bar{E}_{\alpha}^{(p)}=\left\{x: \limsup _{r \rightarrow 0} \frac{\log \gamma_{p}\left(B_{r}(x)\right)}{\log r}=\alpha\right\} .
\end{aligned}
$$

In this paper, we assume that $0 \log 0=0$ for convenience. We define for $r \in[0,1]$

$$
g(r, p)=\frac{r \log p+(1-r) \log (1-p)}{r \log a+(1-r) \log b} .
$$

From now on we will use $g(r, p)$ as the above definition. 


\section{Main results}

From now on, we fix the contraction ratios $a, b$ to construct the self-similar attractor $F$ and assume that $s$ is the real number satisfying $a^{s}+b^{s}=1$.

Proposition 1 ([1, 2]). We have

(1) $\underline{F}(r)=\underline{E}_{g(r, p)}^{(p)}$ if $0<p<a^{s}$,

(2) $\underline{F}(r)=\bar{E}_{g(r, p)}^{(p)}$ if $a^{s}<p<1$,

(3) $\bar{F}(r)=\bar{E}_{g(r, p)}^{(p)}$ if $0<p<a^{s}$,

(4) $\bar{F}(r)=\underline{E}_{g(r, p)}^{(p)}$ if $a^{s}<p<1$.

Further if we put $\delta(r)=g(r, r)$, then

(5) $\underline{F}(r)=\underline{E}_{\delta(r)}^{(r)}$ if $0<r<a^{s}$,

(6) $\bar{F}(r)=\underline{E}_{\delta(r)}^{(r)}$ if $a^{s}<r<1$.

From now on, we set $\delta(r)=g(r, r)$ where $0 \leq r \leq 1$.

Proposition 2 ([6, 3, 10]). For $0 \leq r_{1} \leq r_{2} \leq 1$,

$$
\operatorname{dim}\left(F\left[r_{1}, r_{2}\right]\right)=\inf _{r_{1} \leq r \leq r_{2}} \delta(r),
$$

and

$$
\operatorname{Dim}\left(F\left[r_{1}, r_{2}\right]\right)=\sup _{r_{1} \leq r \leq r_{2}} \delta(r)
$$

Theorem 3. $\operatorname{dim}\left(\cup_{r_{1} \leq r \leq 1} \underline{F}(r)\right)=\operatorname{Dim}\left(\cup_{r_{1} \leq r \leq 1} \underline{F}(r)\right)=\delta\left(r_{1}\right)$ where $a^{s} \leq$ $r_{1} \leq 1$. Similarly $\operatorname{dim}\left(\cup_{0 \leq r \leq r_{2}} \bar{F}(r)\right)=\operatorname{Dim}\left(\cup_{0 \leq r \leq r_{2}} \bar{F}(r)\right)=\delta\left(r_{2}\right)$ where $0 \leq$ $r_{2} \leq a^{s}$.

Proof. We note that it trivially holds for $r_{1}=a^{s}$. For $a^{s}<p<1, g(r, p)$ is a decreasing function for $r \in[0,1]$. We note that

$$
\liminf _{k \rightarrow \infty} \frac{n_{1}(x \mid k)}{k}=r \Leftrightarrow \limsup _{k \rightarrow \infty} \frac{\log \gamma_{p}\left(c_{k}(x)\right)}{\log \left|c_{k}(x)\right|}=g(r, p)
$$

(cf. Lemma 1 in [5]). Hence for $a^{s}<r_{1} \leq 1$, if $r \geq r_{1}$, then $g\left(r, r_{1}\right) \leq g\left(r_{1}, r_{1}\right)$. Therefore if $x \in \cup_{r_{1} \leq r \leq 1} \underline{F}(r)$, then

$$
\limsup _{k \rightarrow \infty} \frac{\log \gamma_{r_{1}}\left(c_{k}(x)\right)}{\log \left|c_{k}(x)\right|} \leq g\left(r_{1}, r_{1}\right)
$$

which means that $\operatorname{Dim}\left(\cup_{r_{1} \leq r \leq 1} \underline{F}(r)\right) \leq g\left(r_{1}, r_{1}\right)=\delta\left(r_{1}\right)$ from the cylinder density theorem in $[2,9]$ and the regular density theorem in the Proposition 2.3 in $[8]$.

It remains to show that $\operatorname{dim}\left(\cup_{r_{1} \leq r \leq 1} \underline{F}(r)\right) \geq \delta\left(r_{1}\right)$. It follows from that

$$
\operatorname{dim}\left(\cup_{r_{1} \leq r \leq 1} \underline{F}(r)\right) \geq \operatorname{dim}\left(F\left[r_{1}, r_{1}\right]\right)=\delta\left(r_{1}\right) .
$$

Similarly $\operatorname{dim}\left(\cup_{0 \leq r \leq r_{2}} \bar{F}(r)\right)=\operatorname{Dim}\left(\cup_{0 \leq r \leq r_{2}} \bar{F}(r)\right)=\delta\left(r_{2}\right)$ where $0 \leq r_{2} \leq a^{s}$ holds from the dual arguments for $0<p<a^{s}$. 
Theorem 4. $\operatorname{dim}\left(\cup_{r_{2} \leq r \leq 1} \bar{F}(r)\right)=\delta\left(r_{2}\right)$ where $a^{s} \leq r_{2} \leq 1$. Similarly $\operatorname{dim}\left(\cup_{0 \leq r \leq r_{1}} \underline{F}(r)\right)=\delta\left(r_{1}\right)$ where $0 \leq r_{1} \leq a^{s}$.

Proof. We note that it trivially holds for $r_{2}=a^{s}$. For $a^{s}<p<1, g(r, p)$ is a decreasing function for $r \in[0,1]$. We note that

$$
\limsup _{k \rightarrow \infty} \frac{n_{1}(x \mid k)}{k}=r \Leftrightarrow \liminf _{k \rightarrow \infty} \frac{\log \gamma_{p}\left(c_{k}(x)\right)}{\log \left|c_{k}(x)\right|}=g(r, p)
$$

(cf. Lemma 1 in [5]). Hence for $a^{s}<r_{2} \leq 1$, if $r \geq r_{2}$, then $g\left(r, r_{2}\right) \leq g\left(r_{2}, r_{2}\right)$. Therefore if $x \in \cup_{r_{2} \leq r \leq 1} \bar{F}(r)$, then

$$
\liminf _{k \rightarrow \infty} \frac{\log \gamma_{r_{2}}\left(c_{k}(x)\right)}{\log \left|c_{k}(x)\right|} \leq g\left(r_{2}, r_{2}\right)
$$

which means that $\operatorname{dim}\left(\cup_{r_{2} \leq r \leq 1} \bar{F}(r)\right) \leq g\left(r_{2}, r_{2}\right)=\delta\left(r_{2}\right)$ from the cylinder density theorem in $[2,9]$ and the regular density theorem in the Proposition 2.3 in [8].

It remains to show that $\operatorname{dim}\left(\cup_{r_{2} \leq r \leq 1} \bar{F}(r)\right) \geq \delta\left(r_{2}\right)$. It follows from that

$$
\operatorname{dim}\left(\cup_{r_{2} \leq r \leq 1} \underline{F}(r)\right) \geq \operatorname{dim}\left(F\left[r_{2}, r_{2}\right]\right)=\delta\left(r_{2}\right) .
$$

Similarly $\operatorname{dim}\left(\cup_{0 \leq r \leq r_{1}} \underline{F}(r)\right)=\operatorname{dim}\left(\cup_{0 \leq r \leq r_{1}} \underline{F}(r)\right)=\delta\left(r_{1}\right)$ where $0 \leq r_{1} \leq a^{s}$ holds from the dual arguments for $0<p<a^{s}$.

\section{Corollary 5.}

$$
\operatorname{dim}\left(\left[\cup_{r_{2} \leq r \leq 1} \bar{F}(r)\right] \cap\left[\cup_{0 \leq r \leq r_{1}} \underline{F}(r)\right]\right)=\min \left\{\delta\left(r_{1}\right), \delta\left(r_{2}\right)\right\}
$$

where $0 \leq r_{1} \leq a^{s} \leq r_{2} \leq 1$.

Proof. It is obvious from the above theorem and the fact that

$$
\operatorname{dim}\left(F\left[r_{1}, r_{2}\right]\right)=\min \left\{\delta\left(r_{1}\right), \delta\left(r_{2}\right)\right\}
$$

which is immediate from Proposition 2.

\section{Corollary 6.}

$$
\operatorname{dim}\left(\left[\cup_{r_{2} \leq r \leq 1} \bar{F}(r)\right] \cap\left[\cup_{0 \leq r \leq r_{1}} \underline{F}(r)\right]\right)=\delta\left(r_{2}\right)
$$

where $a^{s} \leq r_{1} \leq r_{2} \leq 1$. Similarly

$$
\operatorname{dim}\left(\left[\cup_{r_{2} \leq r \leq 1} \bar{F}(r)\right] \cap\left[\cup_{0 \leq r \leq r_{1}} \underline{F}(r)\right]\right)=\delta\left(r_{1}\right)
$$

where $0 \leq r_{1} \leq r_{2} \leq a^{s}$.

Proof. For $a^{s} \leq r_{1} \leq r_{2} \leq 1$,

$$
\operatorname{dim}\left(\left[\cup_{r_{2} \leq r \leq 1} \bar{F}(r)\right] \cap\left[\cup_{0 \leq r \leq r_{1}} \underline{F}(r)\right]\right) \leq \delta\left(r_{2}\right)
$$

from the above theorem.

$$
\operatorname{dim}\left(\left[\cup_{r_{2} \leq r \leq 1} \bar{F}(r)\right] \cap\left[\cup_{0 \leq r \leq r_{1}} \underline{F}(r)\right]\right) \geq \delta\left(r_{2}\right)
$$

since

$$
\operatorname{dim}\left(F\left[r_{1}, r_{2}\right]\right)=\min \left\{\delta\left(r_{1}\right), \delta\left(r_{2}\right)\right\}=\delta\left(r_{2}\right)
$$


which is immediate from Proposition 2. Similarly it holds for $0 \leq r_{1} \leq r_{2} \leq a^{s}$ from the dual arguments.

Remark 1. We note that

$$
\operatorname{Dim}\left(\left[\cup_{r_{2} \leq r \leq 1} \bar{F}(r)\right] \cap\left[\cup_{0 \leq r \leq r_{1}} \underline{F}(r)\right]\right)=s
$$

where $0 \leq r_{1} \leq r_{2} \leq 1$ since $F[0,1] \subset\left[\cup_{r_{2} \leq r \leq 1} \bar{F}(r)\right] \cap\left[\cup_{0 \leq r \leq r_{1}} \underline{F}(r)\right]$ and $\operatorname{Dim}(F[0,1])=s$ from Proposition 2 .

From now on, we denote $H^{s}$ for the $s$-dimensional Hausdorff measure and $A^{c}=F-A$.

Corollary 7. $H^{s}\left(\left[F\left(a^{s}\right)\right]^{c}\right)=0$.

Proof. Let for each $n \in \mathbb{N}$

$$
\bigcup_{a^{s}+\frac{1}{n} \leq r \leq 1} \bar{F}(r) \cup \bigcup_{0 \leq r \leq a^{s}-\frac{1}{n}} \underline{F}(r)=G_{n} .
$$

Then $H^{s}\left(G_{n}\right)=0$ since $\operatorname{dim}\left(G_{n}\right)<s$ for all $n \in \mathbb{N}$ from the above theorem. Since $H^{s}$ is countably sub-additive and $\left[F\left(a^{s}\right)\right]^{c}=\cup_{n \in \mathbb{N}} G_{n}$, it follows.

Remark 2. We note that

$$
\operatorname{dim}\left(\left[F\left(a^{s}\right)\right]^{c}\right)=s
$$

even though

$$
H^{s}\left(\left[F\left(a^{s}\right)\right]^{c}\right)=0 .
$$

Corollary 8. $0<H^{s}\left(F\left(a^{s}\right)\right)<\infty$.

Proof. We see that $0<H^{s}(F)<\infty$ from (2.42) of [8]. We also note that $F\left(a^{s}\right)$ is a Borel set. By the above corollary, it follows since $H^{s}(F)=H^{s}\left(F\left(a^{s}\right) \cup\right.$ $\left.\left[F\left(a^{s}\right)\right]^{c}\right)=H^{s}\left(F\left(a^{s}\right)\right)+H^{s}\left(\left[F\left(a^{s}\right)\right]^{c}\right)$.

Corollary 9. If $F$ is a self-similar attractor, then $H^{s}\left(F\left(a^{s}\right)\right)=1$ where $a^{s}+$ $b^{s}=1$.

Proof. For $1-(a+b)>0$, we note that $H^{s}(F)=1$ since $F$ satisfies the strong separation condition from the Proposition 11.4(a) in [8]. We also note that $H^{1}(F)=1\left(H^{1}\right.$ is the one-dimensional Lebesgue measure, [7]) since $F=[0,1]$ if $1-(a+b)=0$. It follows from the same arguments in the proof of the above corollary.

Remark 3. In the above Corollaries, we note that the normal distribution set $F\left(a^{s}\right)$ in a self-similar attractor $F$ has a full $H^{s}$ measure of $F$.

Remark 4. Using Proposition 1 from the Theorem 1.2 of [10], we easily see that for $0 \leq r_{1} \leq r_{2} \leq 1$

$$
\operatorname{dim}\left(\left[\cup_{r_{1} \leq r \leq 1} \underline{F}(r)\right] \cap\left[\cup_{0 \leq r \leq r_{2}} \bar{F}(r)\right]\right)=\sup _{r_{1} \leq r \leq r_{2}} \delta(r)
$$


and

$$
\operatorname{Dim}\left(\left[\cup_{r_{1} \leq r \leq 1} \underline{F}(r)\right] \cap\left[\cup_{0 \leq r \leq r_{2}} \bar{F}(r)\right]\right)=\sup _{r_{1} \leq r \leq r_{2}} \delta(r),
$$

which implies Theorem 3.

\section{References}

[1] I. S. Baek, Relation between spectral classes of a self-similar Cantor sets, J. Math. Anal. Appl. 292 (2004), no. 1, 294-302.

[2] _ Dimensions of distribution sets in the unit interval, Commun. Korean Math. Soc. 22 (2007), no. 4, 547-552.

[3] _ Dimensions of the subsets in the spectral classes of a self-similar Cantor set, Journal of Applied Mathematics and Informatics 26 (2008), no. 3-4, 733-738.

4] Characteristic multifractal in a self-similar Cantor set, Journal of the Chungcheong Math. Soc. 21 (2008), no. 2, 157-163.

[5] _ Multifractal characterization of the Riesz-Nágy-Takács function, preprint.

[6] I. S. Baek, L. Olsen, and N. Snigireva, Divergence points of self-similar measures and packing dimension, Adv. Math. 214 (2007), no. 1, 267-287.

[7] K. J. Falconer, The Fractal Geometry, John Wiley and Sons, 1990

[8] _ Techniques in Fractal Geometry, John Wiley and Sons, 1997.

[9] H. H. Lee and I. S. Baek, A note on equivalent interval covering systems for packing dimension of $\mathbb{R}$, J. Korean Math. Soc. 28 (1991), no. 2, 195-205.

[10] L. Olsen and S. Winter, Normal and non-normal points of self-similar sets and divergence points of self-similar measures, J. London Math. Soc. 67 (2003), no. 2, 103-122.

Department of Mathematics

Pusan University of Foreign Studies

Pusan 608-738, Korea

E-mail address: isbaek@pufs.ac.kr 